\title{
KINETIC ENERGY OF HIGHLY ELASTIC MEMBRANES
}

\author{
BY \\ M. G. HILGERS (Department of Mathematics and Statistics, University of Missouri-Rolla) \\ AND \\ A. C. PIPKIN ${ }^{\dagger}$ (Division of Applied Mathematics, Brown University, Providence, RI)
}

\begin{abstract}
A theory of elastic sheets with bending stiffness has been proposed in which the strain energy density of the sheet includes a dependence on the second-order derivatives. To study the motion of such sheets, a kinetic energy is required that is accurate to the same order. This is obtained by representing the deformation as a power series in the thickness variable. The lowest-order approximation yields the standard membrane kinetic energy. The next order includes a velocity gradient term. A particularly simple physical interpretation for the additional term is obtained. Furthermore, the matrices involved in this term are shown to possess desirable properties, which can be utilized in future analysis.
\end{abstract}

1. Introduction. The theory of large deformations of thin elastic membranes is based on a strain energy per unit initial area $M\left(\boldsymbol{r}_{, a}\right)$ that depends on the first derivatives of the deformation, $\boldsymbol{r}\left(x_{1}, x_{2}\right)$. Minimum energy techniques using $M$ encounter the undesirable feature of seemingly well-posed problems having no deformation that minimizes the energy functional. This occurs when the physical membrane wrinkles. If the minimum energy principle is applied instead to an energy density of the form

$$
W=M\left(\boldsymbol{r}_{, a}\right)+\alpha Q\left(\boldsymbol{r}_{, a}, \boldsymbol{r}_{, a b}\right)
$$

where $\alpha$ is a positive constant and $Q$ is a quadratic function in the second derivatives, a form of plate theory is obtained [1]. The inclusion of the second-order derivatives introduces a dependence on curvature into the strain energy density thereby giving the membrane some bending stiffness.

The analysis in [1] made no special assumption on the nature of $W$, yet from the work of Ball, Currie, and Olver [2] we know that the $W$ must be quasiconvex in $\boldsymbol{r}_{, a b}$ to ensure the existence of a minimizer. (See [3] for a study of various conditions on energy minimizers.) While we could simply require $Q$ to be quasiconvex in $\boldsymbol{r}_{, a b}$, it was desired to investigate the reasonableness of this assumption. This investigation was reported in

Received March 9, 1995.

1991 Mathematics Subject Classification. Primary 73K10.

${ }^{\dagger}$ Deceased. 
[4] and [5], in which an algorithm is given constructing $M$ and $Q$ from the strain energy per initial volume $\widehat{W}$ for the bulk material from which the membrane is formed. It was shown that $W$ produced in this manner is quasiconvex provided that $\widehat{W}$ satisfies certain stability conditions. It should be noted that this regularization is achieved independently of the size of positive $\alpha$ so that the theory can be placed in a well-posed form by adding a negligible bending energy term.

We continue this development with a theory describing the motion of highly elastic membranes. The first step in developing the dynamics is identifying a kinetic energy density that is accurate to the same order as the strain energy density. Various kinetic energies have been offered in other settings to resolve this issue, many a priori. (See, for instance, deformable surface theory [6] or directed surface theory [7]). Common properties of these kinetic energies include the need for velocity gradient terms and the positivity of various scalar or matrix coefficients. As we did in [4] and [5] for $W$, we intend to identify a class of physically reasonable kinetic energy densities for these highly elastic membranes and examine the properties they possess.

Toward this end, we begin in Sec. 2 with an explanation of our notation, and in Sec. 3 we outline the algorithm by which $M$ and $Q$ can be constructed. These techniques are applied in Sec. 4 to the kinetic energy density for the bulk material. We find that

$$
K=\frac{1}{2} \rho\left(\boldsymbol{v} \cdot \boldsymbol{v}+\beta \boldsymbol{f}_{. t} \cdot \boldsymbol{f}_{. t}\right) .
$$

where $\rho$ is the constant density per unit initial area, $\beta$ is a parameter on the order of the squared thickness of the sheet, and $\boldsymbol{f}$ is a function of $\boldsymbol{r}_{. a}$. Performing the time differentiation on $\boldsymbol{f}$ produces an expression that is quadratic in the velocity gradients and nonlinear in the deformation gradients. This is a more complicated expression for kinetic energy than has been used in related works, but one we believe offers insight into the mechanics of these materials. In Sec. 5, we explore the various minimal requirements on $\boldsymbol{f}$ and see how they restrict its form.

The velocity gradient dependence is a direct consequence of approximating a slab of material as a surface. We will show in Sec. 6 that, at least for reflectionally symmetric materials; the higher-order energy originates from two sources. One is the contribution to the kinetic energy from material particles moving tangentially to the surface in a shearing motion. The other is a component of motion from particles moving away from the surface and thereby changing the sheet's thickness. When we approximate the slab as a surface the implications of thickness are lost, including the kinetic energy contributions from these motion components. Yet these energy contributions are on the same order as those due to the bending stiffness and should be included in our formulation.

While a reasonable addition, the velocity gradient terms cannot be arbitrarily included. The kinetic energy must be positive, for one, as is immediately observed in our case. More subtle is the requirement that the kinetic energy should not introduce unstable behavior. As an example consider Ericksen's paper [7] on plane infinitesimal wave motion in Cosserat plates. He assumes a priori that a certain matrix used in expressing the kinetic energy is positive definite. This allows stability conditions to focus on material properties rather than the plate approximations. In Sec. 7, we show that our kinetic 
energy, while more complicated in form, enjoys the same properties. We conclude with an examination of the particular form the kinetic energy takes when the bulk strain energy density is isotropic and linear elastic.

2. Notation. We consider a flat, thin sheet composed of a homogeneous elastic material that is bounded by the planes $x_{3}= \pm h$ in a system of Cartesian coordinates $x_{A}$ where $A=1,2,3$. This sheet undergoes a deformation such that a particle initially at $x_{A}$ at time $t=0$ moves to the place $\boldsymbol{r}^{*}\left(x_{A}, \tau\right)=r_{i}^{*}\left(x_{A}, \tau\right) \boldsymbol{e}_{i}$ at time $t=\tau$. Here, $\boldsymbol{e}_{i}(i=1,2,3)$ are orthogonal unit vectors. We usually use direct vector and dyadic notation, so that if $\boldsymbol{u}=u_{i} \boldsymbol{e}_{i}$ and $\boldsymbol{v}=v_{i} \boldsymbol{e}_{i}, \boldsymbol{u v}$ is the matrix with components $u_{i} v_{j}$. Where it simplifies matters we write $x_{3}=z$, so that the particle labels are $x_{a}(a=1,2)$ and $z$. In general, upper case letters range over $\{1,2,3\}$ while lower case letters have values $\{1,2\}$.

We form a surface by letting $\boldsymbol{r}\left(x_{a}\right)$ be the average of $\boldsymbol{r}^{*}\left(x_{a}, z\right)$ through the thickness of the sheet. The vectors $\boldsymbol{r}_{, a}$ are tangential to this surface, and the unit normal is given by

$$
\boldsymbol{n}=\boldsymbol{N} /|\boldsymbol{N}|, \quad \boldsymbol{N}=\boldsymbol{r}_{, 1} \times \boldsymbol{r}_{, 2} .
$$

The associated stretch tensor $G_{a b}$ is defined by

$$
G_{a b}=\boldsymbol{r}_{, a} \cdot \boldsymbol{r}_{, b} .
$$

Denote the $2 \times 2$ inverse matrix of $G_{a b}$ as $G^{a b}$. We define the reciprocal base vector $\boldsymbol{r}^{a}$ by

$$
\boldsymbol{r}^{a}=G^{a b} \boldsymbol{r}_{, b}
$$

It follows that

$$
\boldsymbol{r}^{a} \cdot \boldsymbol{r}_{, c}=G^{a b} G_{b c}=\delta_{a c},
$$

which implies that the identity matrix can be expressed as

$$
\boldsymbol{I}=G^{c d} \boldsymbol{r}_{, c} \boldsymbol{r}_{, d}+\boldsymbol{n n} \text {. }
$$

Similarly, any vector $\boldsymbol{v}$ can be expressed in terms of the surface vectors as

$$
\boldsymbol{v}=\boldsymbol{r}_{, a}\left(\boldsymbol{r}^{a} \cdot \boldsymbol{v}\right)+\boldsymbol{n}(\boldsymbol{n} \cdot \boldsymbol{v})=\boldsymbol{r}^{a}\left(\boldsymbol{r}_{, a} \cdot \boldsymbol{v}\right)+\boldsymbol{n}(\boldsymbol{n} \cdot \boldsymbol{v}) .
$$

We suppose that the slab of material has a strain energy $\widehat{W}$ per unit initial volume that is a function of the deformation gradients $r_{, A}^{*}$. The derivative of $\widehat{W}$ with respect to $\boldsymbol{r}_{, A}^{*}$ is the vector

$$
\widehat{\boldsymbol{W}}_{A}=\boldsymbol{e}_{i} \partial \widehat{W} / \partial r_{i, A}^{*}
$$

We further suppose that $\widehat{W}$ is a function of the strains $E_{A B}$ defined by

$$
\boldsymbol{r}_{, A}^{*} \cdot \boldsymbol{r}_{, B}^{*}=G_{A B}=\delta_{A B}+2 E_{A B}
$$

We denote the derivatives of $\widehat{W}$ with respect to the strains as

$$
\widehat{W}_{A B}=\widehat{W}_{B A}=\partial \widehat{W} / \partial E_{A B}
$$

where we conventionally regard $\widehat{W}$ as being expressed symmetrically in $E_{A B}$ and $E_{B A}$. Notation similar to this will be used for functions other than $W$ and should be clear from the context. 
3. Derivation of the sheet energy function. The derivation of the sheet strain energy function is a two-step process $[4,5]$. In the first step, the membrane energy function is defined as

$$
M\left(\boldsymbol{r}_{, a}\right)=\min _{\boldsymbol{r}_{, 3}} 2 h \widehat{W}\left(\boldsymbol{r}_{. a}, \boldsymbol{r}_{.3}\right)
$$

We denote the minimizing value as $\boldsymbol{r}_{, 3}=\boldsymbol{f}\left(\boldsymbol{r}_{, a}\right)$, so that

$$
M\left(\boldsymbol{r}_{, a}\right)=2 h \widehat{W}\left(\boldsymbol{r}_{, a}, \boldsymbol{f}\left(\boldsymbol{r}_{, a}\right)\right)
$$

$\widehat{W}$ must be stationary with respect to $\boldsymbol{r}_{, 3}$ at the minimizing value,

$$
\widehat{\boldsymbol{W}}_{3}\left(\boldsymbol{r}_{. a}, \boldsymbol{f}\left(\boldsymbol{r}_{, a}\right)\right)=\mathbf{0}
$$

so at this value of $\boldsymbol{r}_{, 3}$ there is no stress on the planes $x_{3}=$ constant.

The next step is to estimate the bending energy of the sheet. To do this we expand the deformation in powers of $z$ and $h$ as

$$
\boldsymbol{r}^{*}(\boldsymbol{x}, z, t)=\boldsymbol{r}(\boldsymbol{x}, t)+z \boldsymbol{f}^{*}(\boldsymbol{x}, t)+\left(\frac{1}{2} z^{2}-\frac{1}{6} h^{2}\right) \boldsymbol{g}^{*}(\boldsymbol{x}, t)+O\left(h^{2} z\right)+O\left(z^{3}\right),
$$

where the coefficient of $\boldsymbol{g}^{*}$ is written in a form that has mean zero. Since $\boldsymbol{r}(\boldsymbol{x})$ is the mean of $\boldsymbol{r}^{*}$ through the thickness, we intend for the error terms to have this property also. The requirement is placed on $\boldsymbol{f}^{*}$ and $\boldsymbol{g}^{*}$ that the traction vanish on the surfaces $z= \pm h$, that is, $\widehat{\boldsymbol{W}}_{3}(\boldsymbol{x}, \pm h, t)=\mathbf{0}$. This is accomplished to within an error of $O\left(h^{2}\right)$ by letting

$$
\boldsymbol{f}^{*}=\boldsymbol{f}\left(\boldsymbol{r}_{. a}\right) \quad \text { and } \quad \boldsymbol{g}^{*}=\boldsymbol{F}_{a} \cdot \boldsymbol{f}_{. a} \equiv \boldsymbol{g}
$$

where

$$
\boldsymbol{F}_{b}=\boldsymbol{e}_{i} \boldsymbol{e}_{j} \partial f_{i} / \partial r_{j . b}
$$

These expressions are substituted into $\widehat{W}$, and it is expanded in terms of $z$ and $h$. The sheet strain energy is defined to be

$$
W=\int_{-h}^{h} \widehat{W} d z=M\left(\boldsymbol{r}_{, a}\right)+\frac{h^{2}}{6} Q\left(\boldsymbol{r}_{. a}, \boldsymbol{r}_{, a b}\right)+O\left(h^{4}\right)
$$

with $M$ as in (3.2). The exact form of $Q$ is the subject of much of [5] and requires some explanation.

Writing $Q$ in terms of $M$ we obtain, after some manipulation found in [5], that

$$
Q=\boldsymbol{f}_{, a} \cdot \boldsymbol{M}_{a b} \cdot \boldsymbol{f}_{, b}
$$

Here $\boldsymbol{M}_{a b}$ is the second derivative of $M$ with respect to $\boldsymbol{r}_{, a}$ and $\boldsymbol{r}_{, b}$.

For materials with reflectional symmetry, (3.8) can be further simplified. Reflectional symmetry implies that $\boldsymbol{f}=\phi \boldsymbol{n}$ where $\phi$ is the deformed thickness per unit initial thickness. Equation (3.3), which determines $\boldsymbol{f}$, is equivalent to $\widehat{W}_{3 A}=0$. Under appropriate 
conditions, this implies that $E_{3 A}=0$ which yields $f$ is normal to the surface [4]. Thus $\phi$ is determined as a function of $E_{a b}$ by noting

$$
\widehat{W}_{33}\left(E_{a b}, 0, E_{33}\right)=0 \quad \text { with } \phi^{2}=1+2 E_{33} .
$$

Using the notation

$$
M_{a b}=\partial M / \partial E_{a b}, \quad M_{a b c d}=\partial^{2} M / \partial E_{a b} \partial E_{c d}
$$

and grouping the terms

$$
M_{a b c d}^{*}=M_{a b c d}+M_{a c} G^{b d}
$$

we can express $Q$. Two forms are found to be physically reasonable,

$$
Q=\phi^{2} M_{a b c d}^{*}\left(\boldsymbol{n} \cdot \boldsymbol{r}_{, a b}\right)\left(\boldsymbol{n} \cdot \boldsymbol{r}_{, c d}\right)
$$

and

$$
Q_{M}=\phi^{2} M_{a b c d}^{*}\left(\boldsymbol{r}_{, a b} \cdot \boldsymbol{r}_{, c d}\right),
$$

the difference between the two being that $Q$ is strictly bending energy since $\boldsymbol{n} \cdot \boldsymbol{r}_{, a b}$ are normal curvatures and $Q_{M}$ contains strain gradient terms which do not destabilize the deformation.

4. Kinetic energy. To estimate the kinetic energy, we use the expansion (3.4) in conjunction with the relations (3.5). Denote $\boldsymbol{v}^{*}=\boldsymbol{r}_{, t}^{*}$ and $\boldsymbol{v}=\boldsymbol{r}_{, t}$ so that

$$
\boldsymbol{v}^{*}(\boldsymbol{x}, z, t)=\boldsymbol{v}(\boldsymbol{x}, t)+z \boldsymbol{f}_{, t}(\boldsymbol{x}, t)+\left(\frac{1}{2} z^{2}-\frac{1}{6} h^{2}\right) \boldsymbol{g}_{, t}(\boldsymbol{x}, t)+O\left(h^{2} z\right)+O\left(z^{3}\right) .
$$

The kinetic energy per unit initial volume of the sheet is given by

$$
\widehat{K}=\frac{1}{2} \hat{\rho} v^{*} \cdot v^{*}
$$

into which we substitute (4.1) and integrate through the thickness of the sheet. Here $\hat{\rho}$ is the mass per unit volume of the sheet and is assumed to be constant. This results in

$$
K=\int_{-h}^{h} \frac{1}{2} \hat{\rho} \boldsymbol{v}^{*} \cdot \boldsymbol{v}^{*}=\frac{1}{2} \rho\left(\boldsymbol{v} \cdot \boldsymbol{v}+\frac{h^{2}}{3} \boldsymbol{f}_{, t} \cdot \boldsymbol{f}_{, t}+O\left(h^{4}\right)\right)
$$

where $\rho=2 h \hat{\rho}$ is the mass per unit area of the sheet. Hereafter we will neglect the $O\left(h^{4}\right)$. Set $\beta=h^{2} / 3$ and we have the sheet kinetic energy,

$$
K=\frac{1}{2} \rho\left(\boldsymbol{v} \cdot \boldsymbol{v}+\beta \boldsymbol{f}_{, t} \cdot \boldsymbol{f}_{, t}\right) .
$$

Let us consider the higher-order contribution to the kinetic energy. Observe

$$
\boldsymbol{f}_{, t}=\boldsymbol{F}_{a} \boldsymbol{v}_{, a}
$$

so that

$$
\boldsymbol{f}_{, t} \cdot \boldsymbol{f}_{, t}=\boldsymbol{v}_{, b} \cdot \boldsymbol{C}_{a b} \boldsymbol{v}_{, a}
$$

where $\boldsymbol{C}_{a b}=\boldsymbol{F}_{b}^{\mathrm{T}} \boldsymbol{F}_{a}$ is a function of $\boldsymbol{r}_{, a}$. This expression is quadratic in the velocity gradients and nonlinear in the deformation gradients. Similar expressions have entered into the literature of shell and plate theory before. The theory of deformable surfaces put forth by Balaban, Green, and Naghdi [6] has the term most similar to this, but in their work $\boldsymbol{C}_{a b}$ is a scalar function not a matrix and it depends only on $\boldsymbol{x}$ and $t$ and not the deformation gradient. 
5. Symmetry conditions. We now postulate that the elastic sheet has a kinetic energy of the type given in (4.4). This means that on its most primitive level investigating the motion of the sheet will require specifying $W\left(\boldsymbol{r}_{, a}, \boldsymbol{r}_{, a b}\right)$ and $\boldsymbol{f}\left(\boldsymbol{r}_{, a}\right)$. We do not require that these be derived from a bulk energy density. We have provided these derivations in order to examine the reasonableness of assigning to $W$ and $\boldsymbol{f}$ various properties. Hence, one using this model has a wide latitude of choice for $W$ and $\boldsymbol{f}$. However, certain minimum requirements apply. Those concerning $W$ have been discussed in [1].

We suppose that superimposing an arbitrary rigid rotation $\boldsymbol{R}$ on a given deformation rotates $\boldsymbol{f}$ by $\boldsymbol{R}$ :

$$
\boldsymbol{f}\left(\boldsymbol{R} \boldsymbol{r}_{. a}\right)=\boldsymbol{R} \boldsymbol{f}\left(\boldsymbol{r}_{, a}\right) .
$$

Since $\boldsymbol{f}$ can be expressed in terms of the surface vectors using (2.6),

$$
\boldsymbol{f}=\psi_{a} \boldsymbol{r}_{, a}+\phi \boldsymbol{n},
$$

condition (5.1) implies that $\psi_{a}$ and $\phi$ are invariant under $\boldsymbol{R}$. This follows since $\boldsymbol{R} N=$ $\left(\boldsymbol{R} \boldsymbol{r}_{, 1}\right) \times\left(\boldsymbol{R} \boldsymbol{r}_{, 2}\right)$. We observe that $\psi_{a}$ and $\phi$ enjoy this invariance if and only if they are functions of $G_{a b}$.

$\boldsymbol{f}$ derived from $\widehat{W}$ according to (3.3) possesses the above property. For,

$$
\widehat{\boldsymbol{W}}_{3}=\widehat{W}_{3 A} \boldsymbol{r}_{. A}^{*}
$$

and, since the triad of vectors $\boldsymbol{r}_{. A}^{*}$ must be linearly independent, we conclude that by (3.3)

$$
\widehat{W}_{3 A}\left(E_{C D}^{*}\right)=0
$$

at the minimizing strains $E_{C D}^{*}$. Supposing that $\widehat{W}$ obeys the Legendre-Hadamard condition, we have $\widehat{W}_{3 A 3 B}$ is nonnegative definite. If we further suppose that $\widehat{W}_{3 A 3 B}$ is positive definite, then the inverse function theorem yields that the minimizing strains $E_{3 A}^{*}$ can be expressed in terms of the strains $E_{a b}^{*}$. Hence $\boldsymbol{f} \cdot \boldsymbol{r}_{. a}$ and $\boldsymbol{f} \cdot \boldsymbol{f}$ are functions of the strains $E_{a b}^{*}$. With this and using (2.6) and (2.3), we have

$$
\boldsymbol{f}\left(\boldsymbol{r}_{, a}\right)=G^{a b}\left(\boldsymbol{r}_{. b} \cdot \boldsymbol{f}\right) \boldsymbol{r}_{, a}+\phi \boldsymbol{n}
$$

Setting $\psi_{a}=G^{a b}\left(\boldsymbol{r}_{, b} \cdot \boldsymbol{f}\right)$, we have the desired result.

If it is additionally assumed that the sheet is reflectionally symmetric then $\psi_{a}=0$, as previously discussed. If $\widehat{W}$ is not used to produce $W$ and $\boldsymbol{f}$, we take this as the definition of reflectionally symmetric.

Hence the strain energy density and kinetic energy density are completely determined by specifying two functions $M$ and $\phi$, for a reflectionally symmetric sheet. With these, $Q$ is determined by either (3.12) or (3.13). If the sheet is not reflectionally symmetric, then the entire vector $\boldsymbol{f}$ must be specified, and $Q$ is determined by (3.8). If there is an underlying bulk strain energy density, then $M, f$, and $\phi$ are determined by the techniques in Sec. 3. 
6. The velocity gradient term. When velocity gradient terms appear in a kinetic energy expression, they are often expressed in a fashion similar to $\boldsymbol{v}_{, b} \cdot \boldsymbol{C}_{a b} \boldsymbol{v}_{, a}$. While this is functional, it is difficult to interpret. The kinetic energy term that we propose to use, however, has a relatively simple physical meaning.

To see this, let us restrict attention to a reflectionally symmetric material where $\boldsymbol{f}=\phi\left(\boldsymbol{r}_{, a}\right) \boldsymbol{n}$. We recall that $\phi$ is the deformed thickness of the sheet per unit initial thickness. Observe that

$$
\boldsymbol{f}_{, t}=\phi_{, t} \boldsymbol{n}+\phi \boldsymbol{n}_{, t}
$$

and

$$
\boldsymbol{f}_{, t} \cdot \boldsymbol{f}_{, t}=\boldsymbol{v}_{, b} \cdot \boldsymbol{C}_{a b} \boldsymbol{v}_{, a}=\left(\phi_{, t}\right)^{2}+\phi^{2}\left|\boldsymbol{n}_{, t}\right|^{2}
$$

where we have used $\boldsymbol{n} \cdot \boldsymbol{n}_{, t}=0$.

To understand (6.2) more fully, let us return to expression (4.1) and suppose that we have a reflectionally symmetric sheet. With this, the velocity of a material particle in the slab is

$$
\boldsymbol{v}^{*}(\boldsymbol{x}, z, t)=\boldsymbol{v}(\boldsymbol{x}, t)+z \phi_{, t} \boldsymbol{n}+z \phi \boldsymbol{n}_{, t}+O\left(z^{2}\right)
$$

To the lowest order in the thickness, $\boldsymbol{v}^{*}$ is approximated by the velocity of the mean surface $\boldsymbol{v}$. However, particles not on the mean surface possess a component of velocity normal to the surface. This component of motion is viewed externally as the changing thickness of the slab. By approximating the slab as a surface, however, thickness changes are no longer physically meaningful. To include the kinetic energy contribution from this component of motion of the slab the surface kinetic energy must be modified by the term

$$
\frac{1}{2} \rho \beta(\phi, t)^{2}
$$

Similarly, particles not on the mean surface have a velocity component tangential to it, since $\boldsymbol{n} \cdot \boldsymbol{n}_{, t}=0$. This is viewed externally as a shearing motion through the thickness of the slab. Again, the energy contributed by this motion is lost when the slab is approximated as a surface. In order to include it, the surface kinetic energy must be modified by

$$
\frac{1}{2} \rho \beta \phi^{2}\left|\boldsymbol{n}_{, t}\right|^{2}
$$

When the slab is thin, these higher-order kinetic energy contributions are considered small, and hence often neglected. However, these kinetic energy contributions are on the same order of magnitude as the energy due to the bending stiffness of the material. Therefore, to study the motion of highly elastic membranes it is necessary to account for their effect directly.

7. Matrix analysis. While expressing the higher-order kinetic energy dependence as $\boldsymbol{v}_{, b} \cdot \boldsymbol{C}_{a b} \boldsymbol{v}_{, a}$ provides little physical intuition, it is convenient in other settings, including dynamical stability analysis. In these situations various properties are required, such as symmetry or positive definiteness of either the matrices $\boldsymbol{C}_{a b}$ or the matrix sum $\boldsymbol{C}_{a b} v_{a} v_{b}$. Here $v_{a}$ is any vector satisfying $v_{a}^{2}+v_{b}^{2}=1$. We record some of these properties here for future application. 
Clearly,

$$
\boldsymbol{v}_{, b} \cdot \boldsymbol{C}_{a b} \boldsymbol{v}_{, a}=\boldsymbol{f}_{, t} \cdot \boldsymbol{f}_{, t} \geq 0
$$

so that the kinetic energy contribution cannot be negative, which is a strong property of the matrices $\boldsymbol{C}_{a b}$ by themselves. To proceed further, however, we require some relationships concerning $\boldsymbol{f}$ and its derivatives. By differentiating $\boldsymbol{n} \cdot \boldsymbol{n}=1$ and $\boldsymbol{n} \cdot \boldsymbol{r}_{, b}=0$ we find that

$$
\boldsymbol{n} \cdot \boldsymbol{n}_{, a}=0 \quad \text { and } \quad \boldsymbol{r}_{, b} \cdot \boldsymbol{n}_{, a}=-\boldsymbol{n} \cdot \boldsymbol{r}_{, a b} .
$$

Setting $\boldsymbol{v}=\boldsymbol{n}_{, a}$ in (2.6) yields

$$
\boldsymbol{n}_{, a}=-\boldsymbol{r}^{b}\left(\boldsymbol{n} \cdot \boldsymbol{r}_{, a b}\right) .
$$

Since

$$
\boldsymbol{f}_{, a}=\phi_{, a} \boldsymbol{n}+\phi \boldsymbol{n}_{, a},
$$

(7.3) implies

$$
\boldsymbol{f}_{, a}=\left(\boldsymbol{n} \phi_{b}-\phi \boldsymbol{r}^{b} \boldsymbol{n}\right) \cdot \boldsymbol{r}_{, a b}
$$

where

$$
\phi_{a}=\boldsymbol{e}_{i} \partial \phi / \partial r_{i, a}
$$

From this we have

$$
\boldsymbol{F}_{b}=\left(\boldsymbol{n} \phi_{b}-\phi \boldsymbol{r}^{b} \boldsymbol{n}\right) .
$$

We are now in a position to calculate $\boldsymbol{C}_{a b}$ for reflectionally symmetric materials. Since

$$
\boldsymbol{C}_{a b}=\boldsymbol{F}_{b}^{\mathrm{T}} \boldsymbol{F}_{a}
$$

we have

$$
\boldsymbol{C}_{a b}=\phi_{a} \phi_{b}-\phi^{2} G^{a b} \boldsymbol{n n} .
$$

In general, $\boldsymbol{C}_{a b}$ is not symmetric, even for reflectionally symmetric materials as shown by (7.9). However, $\boldsymbol{C}_{a b} v_{a} v_{b}$ is always symmetric as is seen by setting $\boldsymbol{A}=v_{a} \boldsymbol{F}_{a}$ and observing

$$
\boldsymbol{C}_{a b} v_{a} v_{b}=\left(v_{b} \boldsymbol{F}_{b}^{\mathrm{T}}\right)\left(v_{a} \boldsymbol{F}_{a}\right)=\boldsymbol{A}^{\mathrm{T}} \boldsymbol{A} .
$$

Letting $\boldsymbol{x}$ be an arbitrary vector, we consider the quadratic form

$$
\boldsymbol{x} \cdot\left(\boldsymbol{C}_{a b} v_{a} v_{b}\right) \cdot \boldsymbol{x}=\boldsymbol{x} \cdot\left(\boldsymbol{A}^{\mathrm{T}} \boldsymbol{A}\right) \cdot \boldsymbol{x} .
$$

From this we see

$$
\boldsymbol{x} \cdot\left(\boldsymbol{C}_{a b} v_{a} v_{b}\right) \cdot \boldsymbol{x}=(\boldsymbol{A} \cdot \boldsymbol{x})^{2} \geq 0,
$$

so that $\boldsymbol{C}_{a b} v_{a} v_{b}$ is at least nonnegative definite.

It cannot be positive definite, however, as can be seen from the reflectionally symmetric case. We have

$$
\boldsymbol{C}_{a b} v_{a} v_{b}=\left(v_{a} \boldsymbol{\phi}_{a}\right)\left(v_{b} \boldsymbol{\phi}_{b}\right)-\phi^{2} G^{a b} v_{a} v_{b} \boldsymbol{n n} .
$$

Setting $\boldsymbol{x}=\boldsymbol{n} \times\left(v_{b} \phi_{b}\right)$ yields

$$
\boldsymbol{x} \cdot \boldsymbol{C}_{a b} v_{a} v_{b} \boldsymbol{x}=0
$$


A null direction such as this could lead to dynamical instabilities. If it did, this instability would not be a material property. Rather, it would be a kinematical consequence of the surface approximation. However, as will be seen in [9], the linearized expressions that involve $C_{a b} v_{a} v_{b}$ are summed in such a way as to remove this null direction. In fact, the matrix of concern is of the form $\boldsymbol{I}+\boldsymbol{C}_{a b} v_{a} v_{b}$, which from the above analysis is easily seen to be positive definite. This implies that our kinetic energy does not contribute to unstable behavior, at least for plane waves of infinitesimal amplitude. In essence, the issue of stability rests on the acoustic tensor, as it should.

8. Example. Isotropic small-strain theory. We conclude by examining the kinetic energy for infinitesimal deformations in a sheet composed of an isotropic material. Then

$$
\widehat{W}=\frac{E}{2(1+\nu)}\left[E_{A B} E_{A B}+\frac{\nu}{1-2 \nu}\left(E_{A A}\right)^{2}\right]
$$

where $E$ is Young's modulus and $\nu$ is Poisson's ratio. Equation (3.3) when viewed as

$$
\boldsymbol{W}_{3}=W_{3 B} \boldsymbol{r}_{, B}^{*}
$$

is equivalent to $W_{3 A}=0$. This, in turn, implies

$$
E_{3 a}=0 \quad \text { and } \quad E_{33}=\frac{-\nu}{1-\nu} E_{a a}
$$

Therefore,

$$
\phi^{2}=1+2 E_{33}=1-\frac{2 \nu}{1-\nu} E_{a a} .
$$

We define the displacement $\boldsymbol{u}$ as

$$
\boldsymbol{r}(\boldsymbol{x}, t)=\boldsymbol{x}+\boldsymbol{u}(\boldsymbol{x}, t) .
$$

Since the linearized theory considers $|\nabla \boldsymbol{u}|^{2}$ small, by (8.4) we have

$$
\phi=\sqrt{1-B E_{a a}}=1-\frac{1}{2} B E_{a a}+O\left(|\nabla \boldsymbol{u}|^{2}\right)
$$

with $B=2 \nu /(1-\nu)$ and $\nabla=e_{a} \partial / x_{a}$. Simple calculations show that

$$
\boldsymbol{n}=\boldsymbol{e}_{3}-\boldsymbol{\nabla} u_{3}+O\left(|\nabla \boldsymbol{u}|^{2}\right)
$$

so that

$$
\boldsymbol{f}=\left(1-\frac{1}{2} B E_{a a}\right) \boldsymbol{e}_{3}-\nabla u_{3}+O\left(|\nabla \boldsymbol{u}|^{2}\right) .
$$

Recalling (8.3), we find

$$
\boldsymbol{f}_{, t} \cdot \boldsymbol{f}_{, t} \approx\left[E_{33, t}\right]^{2}+\left|\left(\boldsymbol{\nabla} u_{3, t}\right)\right|^{2} .
$$

It is desirable to compare this with the vibrational plate models that Love [10] uses. Since no velocity gradient terms appear in his formulation, we would need to argue that these terms are negligible for a thin, linear elastic material. However, he introduces dynamics by considering it as an inertial body force and not by approximating the kinetic energy. We could derive an expression that approximates the inertia due to the velocity gradient term; however, comparing it to the acceleration is somewhat difficult. Ultimately, we would be judging the relative size of various effects. As such, any a priori beliefs may be unfounded. 
Acknowledgment. This work was partially supported by a University of Missouri Research Board award. We gratefully acknowledge this support.

\section{REFERENCES}

[1] M. G. Hilgers and A. C. Pipkin, Elastic sheets with bending stiffness, Quart. J. Mech. Appl. Math. 45, 57-75 (1992)

[2] J. M. Ball, J. C. Currie, and P. J. Olver, Null Lagrangians, weak continuity, and variational problems of arbitrary order, J. Funct. Anal. 41, 135-174 (1981)

[3] M. G. Hilgers and A. C. Pipkin, Energy-minimizing deformations of elastic sheets with bending stiffness, J. Elasticity 31, 125-139 (1993)

[4] M. G. Hilgers and A. C. Pipkin, Bending energy of highly elastic membranes, Quart. Appl. Math. 50, 389-400 (1992)

[5] M. G. Hilgers and A. C. Pipkin, Bending energy of highly elastic membranes II, Quart. Appl. Math. 54, 307-316 (1996)

[6] M. M. Balaban, A. E. Green, and P. M. Naghdi, Simple force multipoles in the theory of deformation surfaces, J. Math. Phys. 8, no. 5, 1026-1036 (1967)

[7] J. L. Ericksen, Plane infinitesimal waves in homogeneous elastic plates, J. Elasticity 3, 161-167 (1973)

[8] M. G. Hilgers, Dynamics of elastic sheets with bending stiffness, Quart J. Mech. Appl. Math. (to appear)

[9] M. G. Hilgers, Plane infinitesimal waves in elastic sheets with bending stiffness, Math. Mech. Solids (to appear)

[10] A. E. H. Love, A treatise on the mathematical theory of elasticity, 4th edition, Dover, New York, 1944 